\title{
Epidemiology of toxoplasmosis: role of the tick Haemaphysalis longicornis
}

\author{
Yongzhi Zhou' ${ }^{1}$, Houshuang Zhang ${ }^{1}$, Jie Cao ${ }^{1}$, Haiyan Gong ${ }^{1}$ and Jinlin Zhou ${ }^{1,2^{*}}$
}

\begin{abstract}
Background: Toxoplasma gondii infection is mainly caused by ingestion of water or food that is contaminated with oocysts excreted by cats, or by eating raw meat containing T. gondii tissue cysts. However, oral transmission does not explain the common occurrence of toxoplasmosis in a variety of hosts, such as herbivorous animals, birds, and wild rodents. Little information exists on the maintenance of $T$. gondii parasites in nature and routes of transmission to domestic and wild animal hosts. Therefore, this study evaluated the role of Haemaphysalis longicornis ticks in the epidemiology of toxoplasmosis.

Methods: The real-time polymerase chain reaction (qPCR) technique was used to detect the presence of T. gondii DNA in ticks collected from the field. To observe the amount of dynamic changes of T. gondii in the tick's body and its infectivity, microinjection of green fluorescence parasites was performed. Under laboratory conditions, we evaluated if $\mathrm{H}$. longicornis ticks were infected with T. gondii and their potential to transmit the infection to other hosts using traditional parasitological methods coupled with molecular detection techniques.

Results: The infection rates of $T$. gondii parasites among field-collected adult and nymph $H$. longicornis ticks were $11.26 \%$ and $5.95 \%$, respectively. T. gondii can survive and remain infective in a tick's body for at least 15 days. We found that blood feeding of infected ticks did not transmit T. gondii to hosts, however, ingestion of infected ticks may be a transmission route between ticks and other common hosts.
\end{abstract}

Conclusion: The T. gondii infection in ticks could serve as a reservoir for toxoplasmosis transmission.

Keywords: Tick, Haemaphysalis longicornis, Toxoplasma gondii, Transmission, Epidemiology

\section{Multilingual abstracts}

Please see Additional file 1 for translations of the abstract into the six official working languages of the United Nations.

\section{Background}

Toxoplasma gondii is an obligate intracellular protozoan parasite with a broad host range that includes most bird species and virtually all mammals [1], including humans [2]. It is primarily of medical importance for pregnant women and immunocompromised patients such as those with HIV/AIDS [3, 4]. T. gondii may also show veterinary importance as it is causes toxoplasmosis in domestic

\footnotetext{
* Correspondence: jinlinzhou@shvri.ac.cn

${ }^{1}$ Key Laboratory of Animal Parasitology of Ministry of Agriculture, Shanghai Veterinary Research Institute, Chinese Academy of Agricultural Sciences, Shanghai 200241, China

${ }^{2}$ Jiangsu Co-innovation Center for Prevention and Control of Important Animal Infectious Diseases and Zoonoses, Yangzhou 225009, China
}

animals, e.g. neonatal infections and abortion in sheep and goats [3].

Members of the cat family are definitive hosts of $T$. gondii; sexual reproduction of parasites occurs in the small intestine of cats, resulting in the production of oocysts. Infection is mainly caused by ingestion of water or food that is contaminated with oocysts excreted by cats, or by eating raw meat containing $T$. gondii tissue cysts. Although oral transmission has been regarded as the main route of infection $[4,5]$, it does not explain the common occurrence of toxoplasmosis in a variety of hosts, such as herbivorous animals, birds, and wild rodents. These creatures are improbable to have become infected orally by eating meat or cat feces [6]. Currently, limited information is available on the survival of $T$. gondii in natural settings and its transmission to other warmblooded hosts. These unresolved issues in toxoplasmosis epidemiology need to be addressed. 
Some authors have suggested additional transmission routes of $T$. gondii, including transmission by bloodsucking arthropods [7-10]. Ticks, which are blood-sucking vector arthropods, can carry many kinds of microorganisms while feeding on blood from hosts. However, they may not be able to transmit all of the microorganisms they ingest. DNA of the protozoan parasite $T$. gondii was recently found in field-collected unfed ticks $[7,11]$, and there have also been reports of the presence of $T$. gondii in naturally infected hard ticks $[12,13]$. Suspected cases of human toxoplasmosis were described following tick bites in 1965 [10], and several previous reports have claimed the possibility of experimental transmission with $T$. gondii by ticks $[8,9]$. However, some scientists are doubtful that $T$. gondii infection is able to propagate in bodies of poikilothermic animals $[3,14]$. The dispute is centered on the fact that the possible role of ticks in the transmission of toxoplasmosis might mainly come from limitations in methodology. However with the progress achieved in research techniques, it is possible to determine the role of ticks in the epidemiology of toxoplasmosis.

The tick Haemaphysalis longicornis is distributed mainly in East Asia and Australia [15]. As the dominant tick in China, $H$. longicornis is distributed widely both in the north and south of the country. In this study, we firstly investigated natural $T$. gondii infection in ticks using the real-time polymerase chain reaction (qPCR) technique. We then took the transgenic $\mathrm{RH} /$ green fluorescence protein (GFP) strain of $T$. gondii as the marked parasite to evaluate if our sample of $H$. longicornis ticks was infected with $T$. gondii, as well as the ticks' potential to transmit the infection to other hosts.

\section{Methods}

\section{Detection of $T$. gondii DNA in field-collected $H$. longicornis ticks}

The parthenogenesis tick $H$. longicornis was collected at three sites in the suburbs of Qingdao, Shandong province, China. Ticks were collected between April and October 2012 on three occasions, by flagging. They were then separated into larvae, nymph, and adult ticks, and placed into separate test tubes, where they were stored live until the DNA was extracted. Adults and nymphs were examined singly; larvae were pooled into groups of 20. The total DNA from the tick samples were extracted using a QIAAmp ${ }^{\oplus}$ DNA Mini Kit (Qiagen, MA, USA), according to the manufacturer's instructions. Quality and concentration of the DNA samples were determined using a spectrophotometer (NanoDrop Technologies, DE, USA). The purified DNA was diluted in distilled water for subsequent PCR reactions. To detect $T$. gondii infection in the ticks, the qPCR technique for the 529-bp target of $T$. gondii was carried out, as previously described elsewhere [16]. To confirm the authenticity of the target sequence, partial positive samples of qPCR were selected to amplify the whole 529-bp target by regular PCR followed by sequencing, as previously described [17].

\section{Survival and infectivity of T. gondii in a tick's body}

The transgenic RH/GFP strain of $T$. gondii (kindly provided by Dr. Xuan, Obihiro University of Agriculture and Veterinary Medicine, Obihiro, Japan) was maintained in human foreskin fibroblasts cells cultured in Dulbecco's Modified Eagle Medium (Gibco ${ }^{\circ}$ DMEM), with $10 \%$ of fetal bovine serum(FBS) at $37{ }^{\circ} \mathrm{C}$ in a $5 \% \mathrm{CO}_{2}$ incubator. Purification of tachyzoites was performed, as described previously [18]. Parasites and cultured cell debris were washed several times in cold phosphate-buffered saline (PBS), and the resulting pellets were resuspended in cold PBS and passed through a 27-gauge needle and a $5.0 \mu \mathrm{m}$ pore filter (Millipore Corp., Billerica, MA, USA). The $H$. longicornis ticks were maintained in our laboratory. A single engorged female was used to establish the tick colony. A colony of parthenogenesis $H$. longicornis ticks was initiated from one engorged female collected from a deer at the Shanghai Wildlife Park, China. Ticks were reared in a dark incubator at $25{ }^{\circ} \mathrm{C}$, with $92 \%$ relative humidity and fed on a New Zealand white rabbit. After three generations under laboratory conditions, the tick colony was established at the Shanghai Veterinary Research Institute, Chinese Academy of Agricultural Sciences, Shanghai, China [15].

Parasite injection was performed as previously described with some modifications [19]. The injections were done using $10 \mu \mathrm{L}$ microcapillaries (Drummond Scientific, Broomall, PA, USA) drawn to fine-point needles using a micropipette puller (Narishige, Tokyo, Japan). The needles were then loaded onto a microinjector (Narishige, Tokyo, Japan). T. gondii $\left(10^{3}\right)$ in $0.5 \mu$ l of PBS and $0.5 \mu \mathrm{l}$ of the buffer alone were microinjected from fourth coxae into the haemocoel of unfed adult ticks fixed on a glass slide with adhesive tape in experimental and control groups, respectively. Injected ticks were incubated for three, five, seven, $10,15,20$, and 30 days at $25{ }^{\circ} \mathrm{C}$, and then DNA was purified from pools (10 ticks) of injected ticks for qPCR detection. The 529-bp DNA fragment in $T$. gondii can be used as a quantitative target for parasite numbers $[16,17]$; the relative amount of the target of the 529-bp element of $T$. gondii compared to the tick actin gene (tick house-keeping gene) is regarded as the marker of the number of parasites in ticks. After microinjection, the tick's body lyses were also observed for live parasites using a fluorescence microscope.

Infectivity of $T$. gondii in ticks was confirmed as follows: Ticks, previously injected with $T$. gondii, were homogenized (groups of 10 adults) in $1.5 \mathrm{ml}$ of normal saline $(0.9 \% \mathrm{NaCl})$ containing penicillin and streptomycin. Ticks, previously injected with buffer PBS, were 
homogenized as controls. Mice (BALB/c, eight weeks old) were injected $(0.5 \mathrm{ml} /$ mouse, three mice) intraperitoneally with the tick homogenate in antibiotic solution. Injected mice were observed daily. When clinical symptoms occurred in mice that were killed, such as apathy, depression, and abdominal swelling, appeared, peritoneal dropsy was performed to observe for live parasites using a fluorescence microscope.

Experimentally infecting $H$. longicornis ticks with $T$. gondii To confirm tick infection of $T$. gondii by normal tick feeding, we studied tick infection after ticks fed on the blood of infected mice hosts. BALB/c mice (eight weeks old) were used in all experiments, and inoculations were performed as described previously [20]. For inoculation, tachyzoites were cultured and purified from host cells and washed twice in PBS. Parasites were then diluted in PBS with 4000 tachyzoites of the RH-GFP strain (in $500 \mu \mathrm{l}$ ). Mice inoculated with PBS alone were the negative controls. One day after inoculation with $T$. gondii, about 50 nymphs were allowed to feed to repletion on one infected mouse (there was a total of five mice). Engorged nymphs were allowed to molt in the dark at $25{ }^{\circ} \mathrm{C}, 92 \% \mathrm{RH}$. Newly molted adult ticks were collected and used for T. gondii qPCR detection.

\section{Transmission of $T$. gondii to mice and rabbits by infected ticks}

Adult $H$. longicornis ticks, infected with $T$. gondii by microinjection or by blood feeding, were used in the transmission experiments. Mice and rabbits served as animal hosts. Twenty eight-week-old BALB/c mice were used in the two tick groups (infection via microinjection or by blood-feeding tick). Four adult $H$. longicornis ticks were allowed to feed to repletion on each mouse. Four New Zealand white rabbits ( $2.5 \mathrm{~kg}$ weight) were used for each of the other two tick groups. Forty adult $H$. longicornis ticks were allowed to feed to repletion on each rabbit. One, 10, and 20 days following tick drop-off, blood was collected for qPCR detection, and sera from the individual mice and rabbits were analyzed using recombinant SAG2 with 1:5000 anti-immunoglobulin G (IgG) antibody [21]. One-month post tick drop-off, blood, heart, lung, spleen, liver, brain, and peritoneal fluid of mice and rabbits were taken and divided between microscopic observation for green $T$. gondii and 529-bp based quantitative PCR for $T$. gondii [15]. Mice were used for blind passages, in which the subsequent batches of mice were inoculated with homogenized organs from the sacrificed mice or rabbits [22].

\section{Transmission of T. gondii to mice by ingestion of infected ticks}

$H$. longicornis nymphs, engorged on $T$. gondii infected mice, were collected and allowed to molt to the adult stage. Adult ticks infected with $T$. gondii were homogenized in pools (10 adults), each in $0.5 \mathrm{ml}$ of normal saline $(0.9 \% \mathrm{NaCI})$ containing penicillin and streptomycin. Twenty BALB/c mice (eight weeks old) were force-fed the ticks containing the antibiotic solution homogenate. Mice were observed daily and when clinical symptoms appeared in the mice that were killed, such as apathy, depression, and abdominal swelling, peritoneal dropsy was then performed using a fluorescence microscope. Real-time PCR was performed as the above mentioned method [15].

\section{Animal care and ethics}

The animals used for the experiments were treated following the approved guidelines of the Animal Care and Use Committee of the Shanghai Veterinary Research Institute.

\section{Results}

\section{Detection of $T$. gondii infection in field-collected $H$. longicornis ticks}

The infection rates of $T$. gondii parasites among fieldcollected adult, nymph, and larval $H$. longicornis ticks were $11.26 \%$ (34/302), $5.95 \%$ (5/84), and $0 \%(0 / 36)$, respectively, using qPCR detection. This demonstrates that adult ticks are most likely to be infected with $T$. gondii; meanwhile, no infected larvae were found. Selected positive samples were amplified by regular PCR for the 529bp target. Products were sequenced and were identical to the sequences of the $T$. gondii 529-bp gene that is stored in GenBank.

\section{Survival and infectivity of the T. gondii infection in ticks following microinjection}

The relative amount of the $T$. gondii 529-bp target against the tick actin gene is presented in Fig. 1 . The results indicate that $T$. gondii can survive in a tick's body for extended time periods, but the relative amount of $T$. gondii in ticks seems to show a downward trend from day five to 30 . Over the course of 30 days, the $T$. gondii infection had two peaks (on day five and 15) in H. longicornis ticks. The green fluorescence RH-GFP parasites were observed in the lyses of a tick's body on day three, five, seven, 10, and 15, after microinjection. Figure 2a shows the green fluorescence RH-GFP parasites in the lyses of $H$. longicornis tick's body on day seven, after microinjection.

The infective ability of $T$. gondii in ticks on different days following the injection was tested by inoculating mice intraperitoneally with tick homogenate. T. gondii in $H$. longicornis ticks on day three, five, seven, 10, and 15 after injection infected and killed the mice, but the mice in the control group stayed alive. The green fluorescence RH-GFP parasites were also observed in the peritoneal dropsy of mice inoculated with homogenate of lyses of 


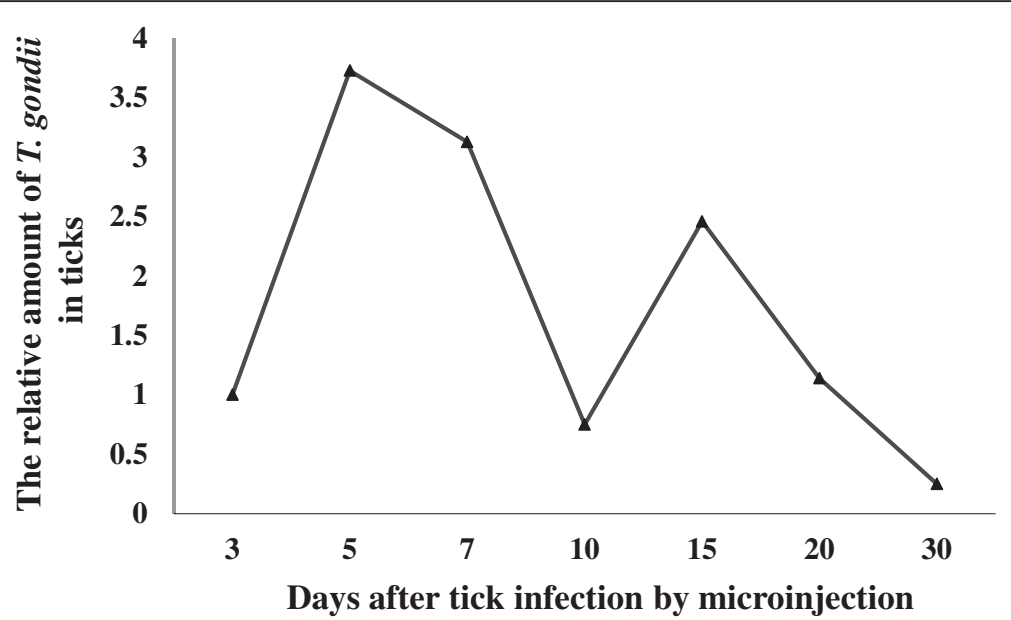

Fig. 1 Variation of the numbers of $T$. gondii in ticks at different times after infection by microinjection. The relative amount of T. gondii in ticks was showed by copies of the 529-bp fragment of T. gondii DNA against copies of the tick actin gene. The $2^{-\Delta \mathrm{Ct}}$ method was used to calculate relative abundance

H. longicornis tick's body on day seven after microinjection (see Fig. 2b).

\section{Experimental infection of ticks with $T$. gondii}

Naturally, ticks take blood from the host animal and then molt into the next stage. To confirm tick infection of $T$. gondii by normal tick blood feeding, we evaluated existent $T$. gondii infection in adult $H$. longicornis ticks, which developed from nymphs feeding on infected mice. The amount of engorged nymphs in each mouse varied from 28 to 34 . Results showed a high infection rate (mean = $38.71 \%$ ) of adult ticks developed from engorged nymphs; the infection rates of tick groups feeding on different mice ranged from $28.57 \%$ to $48.48 \%$ (see Table 1 ). These results demonstrate that $T$. gondii can be transmitted to ticks by ticks feeding on the blood of infected mammal hosts.

\section{Transmission of the $T$. gondii infection to mice and} rabbits by infected tick feeding

Four rabbits for transmission test by ticks infected with microinjection or blood feeding respectively, there is no successful transmission; in the same way, 20 mice for transmission test by ticks infected with microinjection or blood feeding respectively, there is also no successful transmission. Neither in mice nor in rabbit host experiments, were the specific antibodies or nucleotide or green fluorescent parasites detected. Blind passages of mice were taken and did not found positive result.

\section{Transmission of $T$. gondii to mice by ingestion of infected ticks}

In this experiment, 13 of 20 mice were infected based on observation of green fluoresced parasites and qPCR detection in peritoneal dropsy. The seven non-infected mice did not become positive for $T$. gondii in the twomonth post-treatment period. These results indicate that ingesting ticks infected with $T$. gondii can lead to mammals acquiring a natural infection.

\section{Discussion}

H. longicornis ticks are mainly distributed in East Asia and Australia, and transmit a wide range of pathogens including
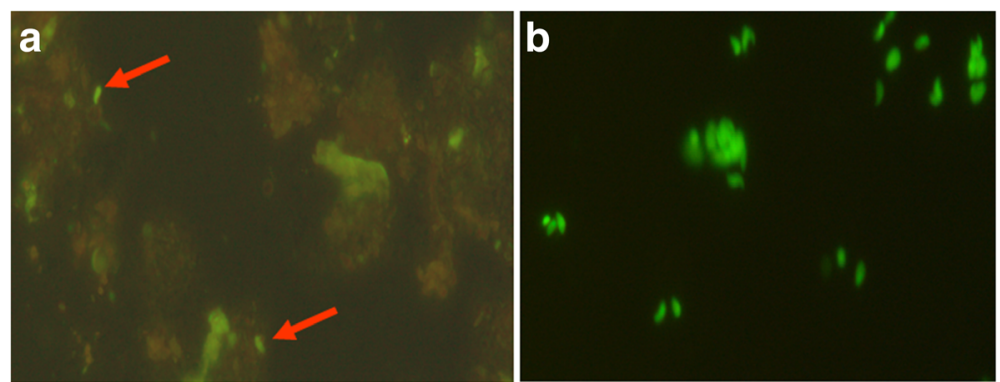

Fig. 2 Survival of T. gondii in tick's body and its infectivity to mice. a: arrows show the green fluorescence RH-GFP parasites observed in the lyses of a H. longicornis tick's body on day seven, after microinjection; $\mathbf{b}$ : green fluorescence RH-GFP parasites in peritoneal dropsy of dead mice inoculated with homogenate of injected ticks 
Table 1 Tick infection by blood feeding in moue infected with T. gondii

\begin{tabular}{llll}
\hline $\begin{array}{l}\text { Mice fro tick } \\
\text { feeding }\end{array}$ & $\begin{array}{l}\text { Number of } \\
\text { ticks }\end{array}$ & $\begin{array}{l}\text { Number of positive } \\
\text { ticks }\end{array}$ & $\begin{array}{l}\text { Positive rate of } \\
\text { ticks }\end{array}$ \\
\hline A & 28 & 11 & $39.28 \%$ \\
B & 33 & 16 & $48.48 \%$ \\
C & 35 & 10 & $28.57 \%$ \\
D & 25 & 8 & $32 \%$ \\
E & 34 & 15 & $44.12 \%$ \\
Total & 155 & 60 & $38.71 \%$ \\
\hline
\end{tabular}

bovine theileriosis (Theileria spp.), bovine babesiosis (Babesia ovata), canine babesiosis (Babesia gibsoni), human rickettsiosis (Rickettsia japonica), and an emerging tick-borne zoonosis (severe fever with thrombocytopenia syndrome) [23-26]. Our study showed that T. gondii DNA were detected in H. longicornis ticks at rates of $11.26 \%$ and $5.95 \%$ in adult and nymph ticks, respectively. This result is similar to the infection rate of $T$. gondii in the Ixodes ricinus tick, which is mainly distributed in Europe [11]. These unfed ticks from the field are derived from molted engorged larva and nymph ticks; therefore they are no contamination from host animal blood. This indicates the natural infection or survival of $T$. gondii in the $H$. longicornis tick. This study found that adult ticks are most likely to be infected with $T$. gondii; meanwhile, no infected larvae were found. The different levels of presence of $T$. gondii at different developmental stages of ticks might be associated with the frequency with which blood-feeding ticks feed.

The survival of the T. gondii infection in H. longicornis ticks was studied by microinjection of a $T$. gondii strain exhibiting green fluorescence. T. gondii can survive and maintain infectivity in $H$. longicornis ticks for at least 15 days, although other tick species may differ. Peaks of the $T$. gondii infection in $H$. longicornis ticks at different times suggest parasite growth, which contrasts with the present theory that $T$. gondii infection propagates only in warm-blooded hosts $[3,27]$. It is necessary to explore this with further experiments.

We validated that ticks infected with $T$. gondii could originate from feeding on the blood of infected mammal hosts under laboratory conditions. This may explain previous observations of ticks naturally infected with $T$. gondii [7, 11-13].

It had been previously claimed that $T$. gondii could be experimentally transmitted to animals by ticks $[8,9]$. However, we did not observe the successful transmission of $T$. gondii by blood-feeding adult ticks. The results of this study suggest that toxoplasmosis might not belong to a tick-borne disease, although transmission by infected larvae and/or nymph ticks cannot be ruled out.

However, ingestion of infected ticks can be an effective method for transmission of certain pathogens. For instance, the apicomplexan protozoan parasite Hepatozoon canis is transmitted by ingestion [28]. The study demonstrated that ticks become infected when they ingest $T$. gondii, which is present in mammalian blood, and retain $T$. gondii following molting. When mammalian hosts ingest an infected tick, the T. gondii infection can be transmitted. This transmission route resembles traditional oral transmission (consumption of raw meat containing infective $T$. gondii) [3, 27]. Oral transmission of ticks may explain the common occurrence of $T$. gondii in a variety of hosts, such as herbivorous animals, wild rodents, and birds [6]. It is important to determine the prevalence of $T$. gondii infection in ticks to establish whether they could represent a significant infection source for toxoplasmosis.

\section{Conclusion}

Our results indicate that $H$. longicornis ticks can harbor viable $T$. gondii parasites, and ingestion of these infected ticks can represent a secondary mechanism of $T$. gondii transmission between ticks and common hosts.

\section{Additional file}

Additional file 1: Multilingual abstracts in the six official working languages of the United Nations. (PDF $381 \mathrm{~kb}$ )

\section{Abbreviations}

FBS: fetal bovine serum; GFP: green fluorescence protein;

IgG: immunoglobulin G; PBS: phosphate-buffered saline; qPCR: real-time polymerase chain reaction.

Competing interests

The authors declare that they have no competing interests.

Authors' contributions

JZ designed the study and participated in the drafting of the paper. $Y Z$ performed the laboratory tests, the data analysis, and wrote the paper. $\mathrm{HZ}$ carried out the detection of parasite DNA in field-collected ticks. HG and JC helped with various aspects of the experiments and paper revising. All authors read and approved the final version of the paper.

\section{Acknowledgments}

We would like to express our sincere gratitude to Prof. Xuenan Xuan, Obihiro University of Agriculture and Veterinary Medicine, Obihiro, Japan, for providing the transgenic RH/GFP strain Toxoplasma gondii. This work was supported by the Special Fund for Agro-scientific Research in the Public Interest of China (grant no: 200903036).

Received: 11 June 2015 Accepted: 15 February 2016

Published online: 20 February 2016

References

1. Dubey JP. History of the discovery of the life cycle of Toxoplasma gondii. Int J Parasitol. 2009;39:877-882.

2. Jones J, Kruszon-Moran D, Wilson M, McQuillan G, Navin T, McAuley JB. Toxoplasma gondii infection in the United States: seroprevalence and risk factors. Am J Epidemiol. 2001;154:357-65.

3. Dubey JP, Beattie CP. Toxoplasmosis of Animals and Man. Boca Raton: CRC Press Inc; 1988.

4. Tenter AM, Heckeroth AR, Weiss LM. Toxoplasma gondii: from animals to humans. Int J Parasitol. 2000;30:1217-58.

5. Sroka J, Wójcik-Fatla A, Dutkiewicz J. Occurrence of Toxoplasma gondii in water from wells located on farms. Ann Agric Environ Med. 2006;13:169-75. 
6. Antolová D, Reiterová K, Dubinský P. Seroprevalence of Toxoplasma gondii in wild boars (Sus scrofa) in the Slovak Republic. Ann Agric Environ Med. 2007;14:71-3.

7. Sroka J, Chmielewska-Badora J, Dutkiewicz J. Ixodes ricinus as a potential vector of T. gondii. Ann Agric Environ Med. 2003;10:121-3.

8. Deryło A, Toś-Luty S, Dutkiewicz J, Umiński J. Participation of Ixodes ricinus L. ticks in the biology and transmission of Toxoplasma gondii. Wiad Parazytol. 1978:24:585-95.

9. Woke PA, Jacobs L, Jones FE, Melton ML. Experimental results on possible arthropod transmission of toxoplasmosis. J Parasitol. 1953;39:523-32.

10. Singh I, Basu SM, Narsimhan D, Sardana DN, Kapila CC, Varma RN, et al. Haemorrhagic disease following tick bites suspected toxoplasmosis. Lancet. 1965;285:834-8

11. Sroka J, Wójcik-Fatla A, Zwoliński J, Zając V, Sawczuk M, Dutkiewicz J. Preliminary study on the occurrence of Toxoplasma gondii in Ixodes ricinus ticks from north-western Poland with the use of PCR. Ann Agric Environ Med. 2008;15:333-8.

12. Gidel R, Provost A. Isolement de Toxoplasma gondii chez des Ixodidés du genre Amblyomma naturellement infectés. Ann Inst Pasteur(Paris). 1965;109:613-6.

13. Gill HS, Nair E, Prakash O. Natural infection of Toxoplasma gondii sought in ticks. Indian J Med Res. 1971;59:1035-8.

14. Jira J, Rosický B. Imunodiagnostika a Epidemiologie Toxoplasmosy. Praque: Academia; 1983 (in Czech).

15. Zhou J, Zhou Y, Cao J, Zhang H, Yu Y. Distinctive microRNAs profiles in the salivary glands of Haemaphysalis longicornis related to tick blood-feeding. Exp App Acarol. 2013:59:339-49.

16. Lin Z, Zhang Y, Zhang H, Zhou Y, Cao J, Zhou J. Comparison of loopmediated isothermal amplification (LAMP) and real-time PCR method targeting a 529-bp repeat element for diagnosis of toxoplasmosis. Vet Parasitol. 2012;185:296-300

17. Homan WL, Vercammen M, De Braekeleer J, Verschueren $\mathrm{H}$. Identification of a 200- to 300-fold repetitive 529 bp DNA fragment in Toxoplasma gondii, and its use for diagnostic and quantitative PCR. Int J Parasitol. 2000:30:69-75.

18. Zhang H, Compaore K, Lee E, Liao M, Zhang G, Sugimoto C, et al. Apical membrane antigen 1 is a crossreactive antigen between Neospora caninum and Toxoplasma gondii, and the anti-NcAMA1 antibody inhibits host cell invasion by both parasites. Mol Biochem Parasitol. 2007:151:205-12.

19. Narasimhan S, Montgomery RR, DePonte K, Tschudi C, Marcantonio N, Anderson JF, et al. Disruption of Ixodes scapularis anticoagulation by using RNA interference. Proc Natl Acad Sci U S A. 2004;101:1141-6.

20. Zhang H, Nishikawa Y, Yamagishi J, Zhou J, Ikehara Y, Kojima N, et al. Neospora caninum: application of apical membrane antigen 1 encapsulated in the oligomannose-coated liposomes for reduction of offspring mortality from infection in BALB/c mice. Exp Parasitol. 2010;125:130-6.

21. Huang X, Xuan X, Kimbita EN, Battur B, Miyazawa T, Fukumoto S, et al. Development and evaluation of an enzyme-linked immunosorbent assay with recombinant SAG2 for diagnosis of Toxoplasma gondii infection in cats. J Parasitol. 2002:88:804-7.

22. Zhou Y, Zhang H, Cao J, Gong H, Zhou J. Isolation and genotyping of Toxoplasma gondii from domestic rabbits in China to reveal the prevalence of type III strains. Vet Parasitol. 2013;193:270-6.

23. Fujisaki K, Kawazu S, Kamio T. The taxonomy of the bovine Theileria spp. Parasitol Today. 1994;10:31-3.

24. Jongejan F, Uilenberg G. The global importance of ticks. Parasitology. 2004; 129:S3-S14.

25. Zhang YZ, Zhou DJ, Qin XC, Tian JH, Xiong Y, Wang JB. The ecology, genetic diversity, and phylogeny of Huaiyangshan virus in China. J Virol. 2012;86:2864-8.

26. Liu Q, He B, Huang SY, Wei F, Zhu XQ. Severe fever with thrombocytopenia syndrome, an emerging tick-borne zoonosis. Lancet Infect Dis. 2014;14:763-72.

27. Montoya JG, Liesenfeld O. Toxoplasmosis. Lancet. 2004:363:1965-76.

28. Baneth G, Samish M, Shkap V. Life cycle of Hepatozoon canis (Apicomplexa: Adeleorina: Hepatozoidae) in the tick Rhipicephalus sanguineus and domestic dog (Canis familiaris). J Parasitol. 2007;93:283-99.

\section{Submit your next manuscript to BioMed Central and we will help you at every step:}

- We accept pre-submission inquiries

- Our selector tool helps you to find the most relevant journal

- We provide round the clock customer support

- Convenient online submission

- Thorough peer review

- Inclusion in PubMed and all major indexing services

- Maximum visibility for your research

Submit your manuscript at www.biomedcentral.com/submit
Biomed Central 